\title{
Proprioception and imitation: on the road to agent individuation
}

\author{
P. Andry ${ }^{2}$, P. Gaussier ${ }^{1,2}$, M. Lagarde ${ }^{2}$, S. Boucenna ${ }^{2}$, L. Hafemeister ${ }^{2}$ \\ (1) IUF, (2) ETIS, ENSEA, Univ Cergy-pontoise, CNRS UMR 8051 \\ F-95000 Cergy-Pontoise \\ \{andry, gaussier, lagarde, boucenna, hafemeister\}@ensea.fr
}

\begin{abstract}
Since Piaget, it is well accepded that higher level cognitive functions are settled on low level sensori-motor associations. In this paper, we will show that different kinds of interactive behaviors can emerge according to the kind of proprioceptive function available in a given sensori-motor system. We will study two different examples. In the first one, an internal proprioceptive signal is avaible for the learning of the visuo-motor coordination between an arm and a camera. An imitation behavior can emerge when the robot 's eye focuses on the hand of the experimenter instead of its own hand. The imitative behavior results from the error minimization between the visual signal and the proprioceptive signal. Here, the imitation results from the perception ambiguity: the robot mistakes its hand with the experimenter hand! In the second example, a robot head has to recognize the facial expression of the human caregiver. Yet, the robot has no visual feedback of its own facial expression. The human expressive resonance will allows the robot to select the visual features relevant for a particual facial expression. As a result, after few minutes of interactions, the robot can imitates the facial expression of the human partner. We will show the different proprioceptive signals used in both examples can be seen as bootstrap mechanisms for more complex interactions. Applied as a crude model of the human, we will propose that these mechanisms play an important role in the process of individuation.
\end{abstract}

\section{Introduction}

With the recent development of robotics, an important effort has been made to build robots able to interact easily with humans. This effort mainly result in (1) researches trying to enhance the robot's control architecture in order to detect and take into account the other (from speech recognition, to the addition of special primitives processing face/body detection and framing and/or emotion recognition) and (2) technics used to enhance the expressiveness and the aspect of the robot. (1) is dedicated to enhance the robot interaction and (2) is dedicated to enhance the human acceptation of the robot, and both approaches intend to facilitate the exchanges between Human and robots. Nevertheless, as underlined by decades of studies in developmental psychology, understanding the roots of communication is still a major issue, and we still not know what are the right 
"properties" that a system must embed in order to detect, exchange, take turn easily as the young infants do, even at a pre-verbal stage.

In this paper, we wish to show that simple perception-action systems initially designed as homeostatic machines, without embedding any "interactive functions", have shown to be the basis of the emergence of higher level behaviors when placed in interactive situations. Interestingly, these emergent behaviors constitute new building blocks favoring at the same time higher level learning and interaction.

We will give two examples, where the architectures are not designed to interact with others. They are only designed to learn new sensory-motor associations when detecting changes in self dynamics (imbalances). When acting in front of the robot, the human is actively modifying the behavioral dynamics of the robot provoking new situations which are learned by the system.

\section{From visuo-motor learning to sequence learning by imitation}

Our model is a perception-action architecture designed to equilibrate 2 kinds of information : Visual information (V), about the external environment and Proprioception information (Prop), i.e information about the self movements that are being done (angle of joints $\phi_{1}, \phi_{2}, \ldots, \phi_{n}$, speed of the joints $\frac{d}{d \phi}$, etc.. of the devices). In order to compute basic behaviors, like tracking, pointing, reaching objects, the architecture is designed like an homeostatic machine (Figure 1 a.). It tries to minimize the differences between the inputs $\mathrm{V}$ and Prop, and the imbalances generate the movements of the system in order to reduce the corresponding error. To do so, V excites a Neural Field (NF) that compute a dynamical attractors centered on the input stimuli [1]. If Prop can be expressed in the same referential as the NF, then it is easy to extract the command to give to the joints of the arm in order to move this latter (and therefore the value of Prop) toward the maximum of the attractor (Figure $1 \mathrm{~b}$.). This read-out [2] mechanism is the value of the derivative of the attractor at the position of prop. Hence, behaviors of the robot are $\mathrm{NF}$ attractors triggered by $\mathrm{V}$ information (representing sensorymotor "target"). Obviously, the read-out mechanisms is only possible if V and Prop can be computed in the same space. In previous work, we have shown that all information can be computed in a simple 2D space (corresponding to the vision produced by a sole CDD camera). To do so, we used a visuo-motor map of neurons, that associate multi-dimensional and redundant information about the joints into a simple 2D "visual" response. The associations are formed during a learning phase: the system produces initial random arm movements in order to learn to equilibrate input $\mathrm{V}$ and Prop information projected on the Visuomotor map. The result is a system that is able to transform motor information $\left(\phi_{1}, \phi_{2}, \ldots, \phi_{n}\right)$ into a simpler 2D space corresponding to the visual one. Then, the movements to reach visual goals is computed in the visual space thanks to the read-out mechanism. The association of an adaptive visuo-motor map with two 1D neural fields can be seen as a simple and global dynamical representation 


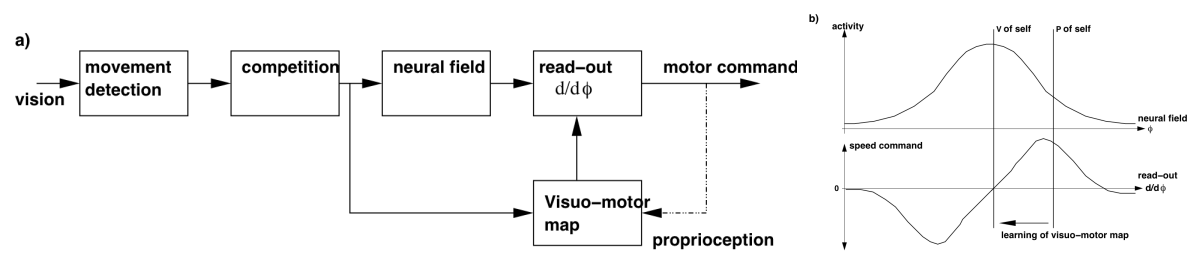

Fig. 1. a. The homeostatic controller trying to equilibrate visual and proprioceptive informations. The motor command is computed by the neural field and the readout groups whose activities are illustrated in b. (example in 1 dimension). In this case Visual and proprioceptive information are different. This unbalance will trigger the learning of a new association on the visuo-motor map.

of the working space controlling efficiently an arbitrary number of degrees of freedom according to 2D information coming from the visual system.

Moreover, having a system that is not able to discriminate visual information about self movements with the movements of others can be, for example, useful to trigger an imitative behavior. Based on movement detection, the system can't differentiate it's extremity from another moving target. As a consequence, moving in front of the robot induces visual changes (movement detection) that the robot interprets as an unforeseen self movement. The robot acts as an homeostat, it tends to correct by producing the opposite movements, inducing the following of the demonstrator gesture (numerous psychological works show comparable human behaviors when visual perception is ambiguous [3-5]). Applied to an eyearm robotic system, the generated error will induce movements of the robotic arm reproducing the moving path of the human hand: an imitative behavior emerges. Using this setup, we showed that our robot can imitate several kind of movements (square or circle trajectories, up and down movements. Hence,
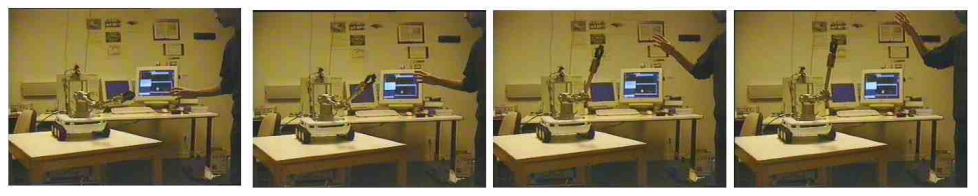

Fig. 2. The proto-imitation principle applied to a robotic setup.

the imitative behavior emerges as a side effect of the perception ambiguity (and limitations). From this low-level imitative behavior, our robot is able to learn sequences of movements from others. To do this, we use a second sensori-motor loop (Figure 3 a.) based on a neurobiological model [6] inspired from some of the properties of the cerebellum and the hippocampus. This model uses associative learning rules between past inputs memorized as a STM and present inputs in order to learn the timing of simple sequences. In order to learn complex sequences 
in which the same state is repeated several times, we have added a mechanism that generates internal dynamics and that can be associated with the repeated inputs of the sequence. The association between the repeated inputs and different activities of the oscillator allows to code hidden states with different and un-ambiguous patterns of activities. As a result, our architecture manages to learn/predict and reproduce complex temporal sequences.

a.

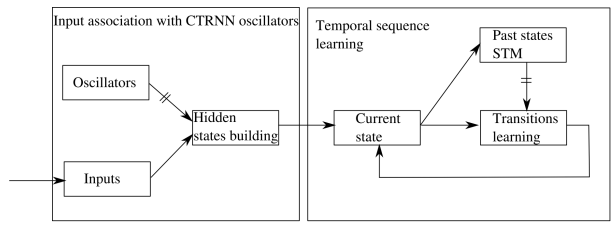

b.

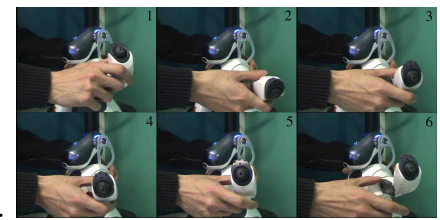

c.

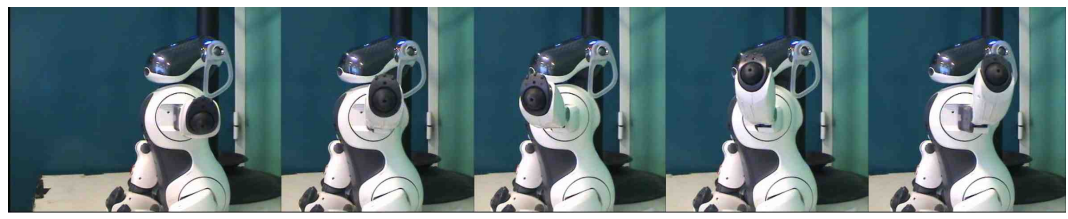

Fig. 3. a. : Complex sequences learning model b. : We manipulate Aibo passively. It learns the succession of orientations of the movement from these front left leg motor information. c. : Aibo reproduces the learnt sequence.

Moreover, recent experiments shows that this architecture can be transposed to learning by demonstration [7], and also to the learning of complex sequences of displacements with a navigating robot [8]. The coupling between the experimenter and the robot is performed via the visual environment (see also [9] for a similar dynamical coupling)but the visuo-motor coupling has been learned thanks to the proprioceptive signal.

\section{Robot response to an expressive human}

This second work is motivated by the question of how a robotic system (Figure 4, right), able to exhibit a set of emotional expressions can learn autonomously to a associate theses expressions with those of others. Here, "autonomously" refers to the ability to learn without the use of any external supervision. A robot with this property could therefore be able to associate its expressions with those of others, linking intuitively its behaviors with the responses of the others. This question is close to the understanding of how babies learn to recognize the facial expressions of their caregivers without having any explicit teaching signal allowing to associate for instance an "happy face" with their own internal emotional state of happiness.

Using the cognitive system algebra [10], we showed that a simple sensori-motor architecture (see Figure 4, left) using a classical conditioning paradigm could 
solve the task if and only if we suppose that the baby produces first facial expressions according to his/her internal emotional state and that next the parents imitate the facial expression of their baby allowing in return the baby to associate these expressions with his/her internal state [11]. If the adult facial expressions are not synchronized with the baby facial expression, the task cannot be learned. Moreover, recent psychological experiments [12] have shown that humans "reproduce" involuntary a facial expression when observing and trying to recognize it. Interestingly, this facial response has also been observed in presence of our robotic head. This low level resonance to the facial expression of the other can be considered as a natural bootstrap for the baby learning ("empathy" from the parents). Because, the agent representing the baby must not be explicitly supervised, a simple solution is to suppose the agent representing the parent is nothing more than a mirror. We obtain an architecture allowing the robot to learn the "internal state"-"facial expression" associations.

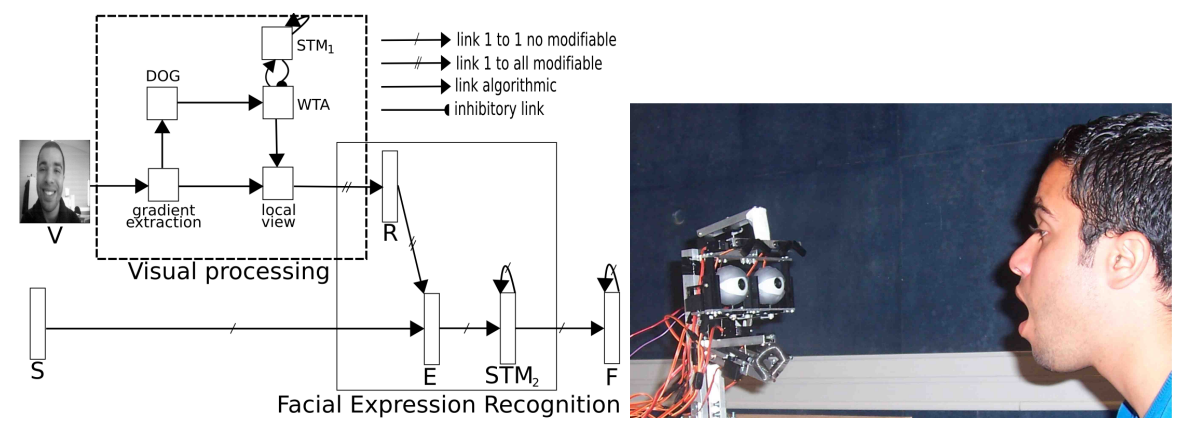

Fig. 4. The architecture used to associate a collection of feature points extracted from the visual flow with the expressed emotion by the robot. If a human come in front of the robot an start to imitate the expression, (s)he will closes the loop between vision and proprioception and allow the system to learn to recognize emotions of others.

We showed that from our departure control architecture, learning is only possible if the parent agent (supposed to be the teacher) imitates the baby agent. The roles are switched according to the classical point of view of AI and learning theory. This advocates that taking account of the interaction dynamics between two agents can change our way of thinking learning and more generally cognition problems.

\section{Discussion}

Both examples show the importance of the interaction with a partner for the robot development and learning. Imitation appears as an important mechanism both for learning and communication [13]. Interestingly, in our developmental 
procedure there is no need at first to suppose the robot is able to recognize the human partner as a model. At the opposite, the robot development is based on the perception ambiguity. The robot considers the human partner as a part of itself. The interaction and the associated correlations extend the proprioception of the robot to the human partner. They become a single dynamical system able to learn things that the robot could not learn by itself. Hence imitative behaviours would be the result at first of the agent unability to perceive something as being different from itself. We can imagine the capability to predict the feedback signal can be used to verify if there is some novelty in the sensory feedback related to the current robot action. Bounded novelty may result from the interaction with a partner hence allowing to recognize him/her at such. Hence we can propose a scheme in which the individuation comes after a phase of fusion/merging with the environement. The proprioceptive signal is necessary to close the loop and to build a global system composed of the agent and his/her caregivers allowing simple learning mechanisms to be sufficient for the learning of more and more complex behaviors (autopoietic loop). First correlations detections allow to build new predictive signals allowing then to differentiate the agent "inner part" from the outside and latter the other agents. We have shown for instance that the capability to discreminate faces from non faces could be the result of the emotional interaction and not one of its preriquisites as usually supposed in classical image processing works. In the same vein, joint attention to a given object could be the result of the emotional association between the facial expression of the experimenter and an object (social referencing) and not the opposite as it is classically supposed. Social interactions can be seen in the first periods of the development as a way to increase the potential of the proprioceptive function allowing to maintain more complex and stable dynamics that can be used for the learning of more complex tasks. Hence, in an epigenitic approach, the sensori-motor control appears as an essential element to avoid the symbol grounding problem and to build autonomous robots able to develop more and more cognitive capabilities thanks to the social interactions.

\section{Acknowledgments}

This work was supported by the French Region Ile de France, the Institut Universitaire de France (IUF) and the FEELIX GROWING european project. (FP6 IST-045169)

\section{References}

1. Amari, S.: Dynamic of pattern formation in lateral-inhibition type by neural fields. Biological Cybernetics 27 (1977) 77-87

2. Schöner, G., Dose, M., Engels, C.: Dynamics of behavior: theory and applications for autonomous robot architectures. Robotic and Autonomous System 16(2-4) (December 1995) 213-245

3. Nielsen, T.: Volition: A new experimental approach. Scandinavian Journal of Psychology 4 (1963) 225-230 
4. Fourneret, P., Jeannerod, M.: Limited conscious monitoring of motor performance in normal subjects. Neuropsychologia 36 (1998) 1133-1140

5. Jeannerod, M.: To act or not to act. perspectives on the representation of actions. Quaterly Journal of Experimental Psychology 52A (1999) 1-29

6. Gaussier, P., Moga, S., Banquet, J.P., Quoy, M.: From perception-action loops to imitation processes. Applied Artificial Intelligence (AAI) 1(7) (1998) 701-727

7. Lagarde, M., Andry, P., Gaussier, P.: The role of internal oscillators for the oneshot learning of complex temporal sequences. In de Sa, J.M., Alexandre, L.A., Duch, W., Mandic, D., eds.: Artificial Neural Networks - ICANN 2007. Volume 4668 of LNCS., Springer (2007) 934-943

8. Lagarde, M., Andry, P., Gaussier, P., Giovannangeli, C.: Learning new behaviors : Toward a control architecture merging spatial and temporal modalities. In: Workshop on Interactive Robot Learning - International Conference on Robotics: Science and Systems (RSS 2008). (June 2008) To appear.

9. Cheng, G., Kuniyoshi, Y.: Complex continuous meaningful humanoid interaction: A multi sensory-cue based approach. In: Proceedings of IEEE International Conference on Robotics and Automation (ICRA2000). (April 2000) 2235-2242

10. Gaussier, P.: Toward a cognitive system algebra: A perception/action perspective. In European Workshop on Learning Robots (EWRL). (2001) 88-100

11. P. Gaussier, K. Prepin, J.N.: Toward a cognitive system algebra: Application to facial expression learning and imitation. In Embodied Artificial Intelligence, F. Iida, R. Pfeiter, L. Steels and Y. Kuniyoshi (Eds.) published by LNCS/LNAI series of Springer (2004) 243-258

12. Nadel, J., Simon, M., Canet, P., Soussignan, R., Blancard, P., Canamero, L., Gaussier, P.: Human responses to an expressive robot. In: Epirob 06. (2006)

13. Nadel, J., Potier, C.: Imiter être imité dans le développement de l'intentionnalité. Imiter pour découvrir l'humain, Paris, PUF (2002) 83-104 